\title{
Comparison of Different Light Sources
}

\author{
Rajat Kumar Chaudhary ${ }^{1}$, Manish Kumar ${ }^{2}$ \\ UG Student, EEE Department, MAIT/GGSIPU, India ${ }^{1,2}$
}

\begin{abstract}
This paper deals with the comparison of cost saving, energy use and energy saving by fluorescent tubes, incandescent lamps, compact fluorescent lamps and light emitting diodes keeping illumination constant. LEDs are comparatively recent in the field of lighting technology however they can replace older technology due to the advantages offered by them such as the parameters of cost and power saving and energy consumption
\end{abstract}

Keywords: CFL, cost saving, energy consumption, energy saving, LED.

\section{INTRODUCTION}

Different sources of lights such as CFLs, LEDs, fluorescent tube lights, incandescent bulbs are used as lighting sources in different places. However all of these sources have different performances based on power consumption initial investment, total cost etc. If the total illumination level is changed the sources cannot be compared for performance. And hence for all the calculations the illumination is considered to be constant.

The purpose of the paper is to compare different lighting sources based on their construction, working, principle and also on performance and thus establish which source would be the best possible for usage in today's time.

Phenomenon of falling of light on any surface by which visual sensation is observed by human eye is called illumination. Illumination is the reason due to which any surface look more or less bright. Illumination depends on the distance between the light source and the surface on which light is falling. The amount of light falling on a surface is measured in unit called lux. Illumination is measured in foot candles.

Two laws are given for the calculation of illumination on any surface:-

(a) Law of inverse squares - According to this law illumination at any surface is inversely proportional to the squares of distance between the source and the light source treating source as a point source.

(b) Lambert's cosine law- According to this law the illumination at any point on the surface is directly proportional to the cosine of the angle between the normal at that point and the direction of luminous flux.

\section{ENERGY EFFICACY}

The energy efficacy is given by lumen/watt for the lighting system. Higher the energy efficacy better is the system.

\begin{tabular}{|c|c|c|c|}
\hline \begin{tabular}{|c|c|c|}
\hline Light Output: LED vs Incandescent vs CFL \\
\hline
\end{tabular} & $\begin{array}{c}\text { Light Emitting } \\
\text { Diodes (LEDs) }\end{array}$ & $\begin{array}{c}\text { Incandescent } \\
\text { Light Bulbs }\end{array}$ & $\begin{array}{c}\text { Compact } \\
\text { Fluorescents (CFLs) }\end{array}$ \\
\hline Lumens & Watts & Watts & Watts \\
\hline 450 & $4-5$ & 40 & $9-13$ \\
\hline 800 & $6-8$ & 60 & $13-15$ \\
\hline 1,100 & $9-13$ & 75 & $18-25$ \\
\hline 1,600 & $16-20$ & 100 & $23-30$ \\
\hline 2,600 & $25-28$ & 150 & $30-55$ \\
\hline
\end{tabular}

NOTE: The above values are merely indicative and must not be taken too rigidly because the lighting technology is

Figure 1

\section{INCANDESCENT LIGHT BULBS}

\section{A. Construction}

The lamp has a filament and two lead wires . The lead wires are connected to the foot contact and glass support of the bulb individually while the filament is attached across them. For the support of the filament in the middle portion two support wires are also used. Insulation is given between the foot contact and the metallic base the glass bulb protects the filament from oxidation and is either kept as a vacuum or filled with inert gases. 


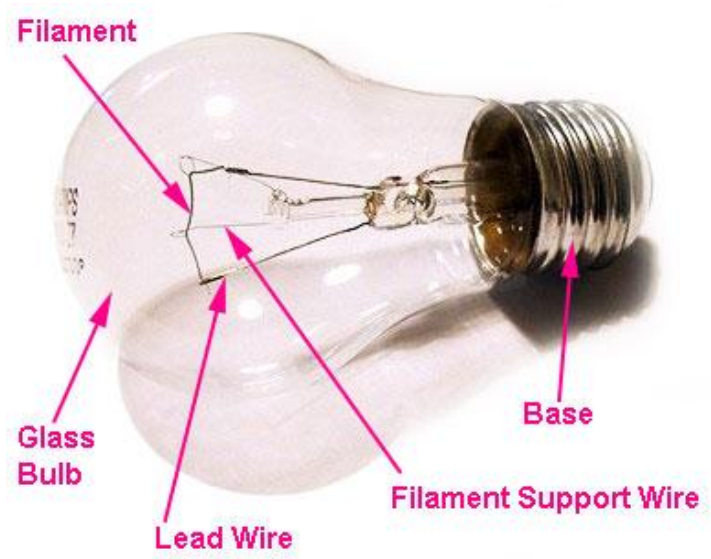

Figure 2

B. Working

The valence electrons of any atom can be excited in a number of ways .Thermal excitation is one such method .If an object is given heat; the electrons in the outer orbit of such an atom would get excited and jump to a higher energy level. However, they cannot stay in such excited states for long. There are certain stable states that an electron acquires. The electron falls down to such a state this leads to loss of energy in the form of photons. The photon is in the form of electromagnetic radiation. The part of this radiation with wavelength in visible region gives visible light. Incandescent means producing visible light by heating an object. When electric current flows through a fine filament, it raises its temperature so that we obtain light.

\section{CFL}

\section{A. Construction}

CFLs are more energy efficient than conventional tube lights and bulbs .Major components of CFL:

1. Electronic ballast circuitry

2. A glass tube which has a coating of fluorescent material and is filled with inert gas and traces of mercury vapor

Parts of ballast:
1. EMI filter
2. Rectifier and smoothing capacitor
3. Control IC
4. Half bridge inverter
5. Resonant tank circuit

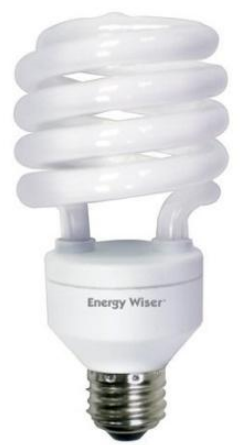

Figure 3

B. CFL working

A current is needed to preheat the filament and high voltage is required for ignition whereas high frequency is required for running process.

To ensure that these conditions are satisfied initially an AC to DC conversion is done at the output .The EMI filter is given to filter out the noise. First the AC voltage is converted to DC by full wave rectifier; the capacitor gives a smooth DC voltage output. A half bridge switching circuit is used so that this DC can be changed into high frequency A.C. It 
has a duty cycle of 50\%. This high frequency AC drives the resonant tank circuit and gives sinusoidal current and voltage at the lamp.

\section{FLUORESCENT TUBE}

\section{A. Construction}

A fluorescent lamp has a long discharge tube. This tube is sealed at low pressure and has two filament electrodes at its opposite ends. It is filled with an inert gas inside and the inner surface is coated with phosphorus. The filaments preheat the tube and help in conduction. This requires a lot of power in the beginning. The inert gas inside, argon for instance also contains a trace of mercury. These mercury atoms change the state to gaseous form due to energy. The collision of these atoms increases the energy even more. When the electrons return to their normal states form the excited state, energy is released .This is in the UV spectrum and not visible to us. The phosphorus coating of the tube when irradiated by UV light makes it emit a white light which we can see. Once conduction between the electrodes is complete, no more heating is required.

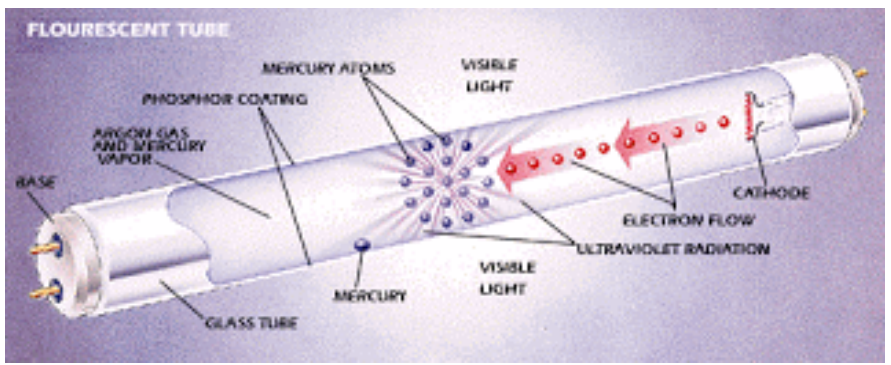

Figure 4

\section{B. Principle}

The process of conversion of electrical energy to light energy in tube lights depends on the inelastic electron collision. When a free electron collides with an atom in the gas, it imparts its energy to the atom's electron which resides in its outer orbit. This causes the electron to gain kinetic energy temporarily. When this electron falls back to its original state it emits an UV photon. This is because most of the photons released by mercury atoms have wavelengths in UV region. The interior coating of the lamp absorbs these UV photons and another emission of photon takes place due to a similar energy jump as before. This photon has lower energy and falls in visible region. The energy lost is used for heating the phosphor coating.

\section{LED}

\section{A. Construction}

The LED has two terminals namely the anode and cathode. The p-n junction is protected by a dome type hemispherical structure which is made of hard plastic epoxy resin. This protects the LED from vibrations or impact. The domed top of the LED helps in concentration of the amount of light. The pin nearest the flat end on outer surface of LED is the cathode (GND) while the other terminal is positive (+).

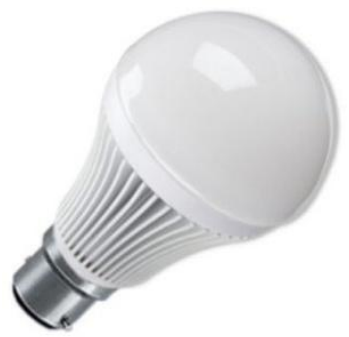

Figure 5

\section{B. Principle}

LEDs work on the principle of recombination. When it is forward biased the free electrons from $\mathrm{n}$ side and holes from $\mathrm{p}$ side move towards the junction where recombination takes place. This also reduces the width of depletion layer. Hence conduction becomes even easier due to higher mobility of electrons; their energy level is higher than that of holes. When recombination takes place, some energy loss is definite due to difference in energy levels of electrons and holes. This energy is released in the form of heat and light. 
C. Working

A LED is like any normal p-n junction diode that conducts when it is forward biased .The application of external voltage across the LED provides the valence electrons with energy.This enables these electrons to break the bond with its parent atom. After breaking bond with its parent atom, the valence electron is called a free electron. The free electrons lie in an energy level called conduction band while the valence electrons lies in an energy level called valence band $^{[1]}$. When the free electrons in the conduction band lose energy, they recombine with the holes in valence band. The hole was created due to the escaping of free electron when energy was provided. This recombination leads to emission of light.

\section{HYPOTHETICAL CASE STUDY OF ACADEMIC BUILDING}

Comparison of elements considered:

Table 1

\begin{tabular}{|l|l|l|l|}
\hline \multicolumn{1}{|c|}{ Element } & \multicolumn{1}{c|}{ Illumination } & \multicolumn{1}{c|}{ Power } & \multicolumn{1}{c|}{ Cost } \\
\hline Fluorescent Tube light & $100 \operatorname{lux}$ & $40 \mathrm{~W}$ & Rs. 45 \\
\hline C.F.L & $30 \mathrm{lux}$ & $15 \mathrm{~W}$ & Rs. 40 \\
\hline Bulb & $85 \mathrm{lux}$ & $100 \mathrm{~W}$ & Rs. 10 \\
\hline LED & $155 \mathrm{lux}$ & $18 \mathrm{~W}$ & Rs. 470 \\
\hline
\end{tabular}

No. of blocks of college considered: 5

Average no. of tube lights per block: 190

1. No. of tube lights $=190 * 5=950$

Cost of system initial installment $=$ Rs. $950 * 45=$ Rs. 42750

Power required $=950 * 40 \mathrm{~W}=38 \mathrm{~kW}($ in $1 \mathrm{hr})=38 \mathrm{kWh}$

Power spent in a day $=38 \mathrm{kWh} * 8=304 \mathrm{kWh}$

(Assuming lights are on for 8 hours on a normal working day)

Power used in a month $=304 * 22=6688 \mathrm{kWh}$

(Assuming 22 working days per month i.e. excluding Saturdays, Sundays)

Power used yearly $=6688 * 12=80256 \mathrm{kWh}$

For initial installment:

1. No. of LEDs required for same level of illumination

$=(100 / 155)^{*}$ no. of TLs

$=(100 / 155) * 950=613$

Cost per LED= Rs. 470

Cost of system $=$ Rs $470 * 613=$ Rs. 288110

2. No. of CFLs required for initial installment $=(100 / 30) * 950=3167$

Cost of initial installment $=$ Rs. $3167 * 40=$ Rs. 126680

3. No. of Bulbs required for initial installment $=(100 / 85) * 950=1185$

Cost of initial installment $=$ Rs. $1118 * 10=$ Rs. 11180

Initial Costs:

Table 2

\begin{tabular}{|l|l|}
\hline \multicolumn{1}{|c|}{ SOURCE } & \multicolumn{1}{c|}{ COST } \\
\hline LED & 288110 \\
\hline Fluorescent Tube Light & 42750 \\
\hline CFL & 126680 \\
\hline Bulb & 11180 \\
\hline
\end{tabular}

Yearly Power consumption:

1. $\mathrm{LED}=613 * 18 * 8 * 22 * 12=23303.81 \mathrm{kWh}$

2. $\mathrm{CFL}=3167 * 15 * 8 * 22 * 12=100330.5 \mathrm{kWh}$

3. Fluorescent Tube Light $=80256 \mathrm{kWh}$

4. $\mathrm{Bulb}=1118 * 100 * 8 * 22 * 12=236121 \mathrm{kWh}$

Assuming cost of electricity as Rs.7 per unit 
Yearly cost of different systems:

1. $\mathrm{LED}=23303.81 * 7=$ Rs. 163112.67

2. Fluorescent tube light $=80256 * 7=$ Rs. 561792

3. $\mathrm{CFL}=100330 * 7=$ Rs. 702310

4. Bulb $=236121.7 * 7=$ Rs. 1652852

Table 3

\begin{tabular}{|l|l|l|l|l|}
\hline \multicolumn{1}{|c|}{ Element } & \multicolumn{1}{c|}{ Power } & \multicolumn{1}{c|}{ Initial cost } & \multicolumn{1}{c|}{ Electricity charge } & \multicolumn{1}{c|}{ Total cost } \\
\hline LED & $23303.81 \mathrm{kWh}$ & 288110 & 163112 & 451222 \\
\hline CFL & $100330 \mathrm{kWh}$ & 126680 & 702310 & 828990 \\
\hline Tube light & $80256 \mathrm{kWh}$ & 42750 & 561792 & 604542 \\
\hline Bulb & $236121.7 \mathrm{kWh}$ & 11180 & 1652852 & 1664032 \\
\hline
\end{tabular}

\section{SOME DISADVANTAGES OF LEDS}

(a). LEDs must be supplied with the correct voltage and current at a constant flow. This requires some electronics expertise to design the electronic drivers.

(b). Due to age and temperature LED's can shift color. And two different LED's will have two different color characteristics, which affect how the light is perceived.

\section{A. Temperature dependence:}

LED performance mainly depends on the ambient temp of the operating environment. Over-driving the LED in high ambient temperatures may result in overheating of the LED package, eventually leading to device failure. For maintaining long life adequate heat-sinking is required. This is especially important when considering automotive, medical and military applications, where the device must operate on a large temperature range, and is required to have a low failure rate.

The efficient use of high brightness LEDs means less heat buildup, coupled with efficient thermal management ensuring optimum performance ${ }^{[2]}$.

B. Light quality:

Most cool-white LEDs have spectra that are different from a black body radiator like the sun or an incandescent light. The spike at $460 \mathrm{~nm}$ and dip at $500 \mathrm{~nm}$ can cause the color of objects to be perceived differently under cool-white LEDs illumination than sunlight or incandescent sources, due to metamerism; red surfaces are rendered particularly by phosphor cool-white LEDs. On the other hand, the color-rendering properties of common fluorescent lamps are often poor in quality to what is now available in state-of-art white LEDs.

The latest LEDs are used by Luminanz and light is mixed within the fitting before it comes out of the fitting which enables us to produce all types of color temperature and color rendering.

\section{Area light source:}

LEDs do not approximate a "point source" of light, rather a lambertian distribution. So it is not used in applications requiring a spherical light field. LEDs are not able to provide divergence below a few degrees. This is contrasted with lasers, because it can produce beams with divergences of 0.2 degrees or less.

The solution to this issue has been found and spherical light fields can be produced ${ }^{[3]}$ and adjustment of beam and spread angles to suit required application can be done.

\section{Blue hazard:}

There is increasing concern that blue LEDs and cool-white LEDs are now able to exceed safe limits of blue-light hazard, as defined in eye safety specifications such as ANSI/IESNA RP-27.1-05: Recommended Practice for Photo biological Safety for Lamp and Lamp Systems.

LED light is used indirectly in Luminanz's technology; which avoids the contact with eye and providing a safe and efficient light output.

E. Blue pollution:

Because cool-white LEDs emit much more blue light than conventional light sources such as high-pressure sodium lamps, the wavelength dependence of Rayleigh scattering means that cool-white LEDs are responsible for more light pollution than any other light sources ${ }^{[4]}$. It is therefore very important to shield the cool-white LEDs fully when used outdoors $^{[5]}$. 
The $460 \mathrm{~nm}$ emission spike of cool-white and blue LEDs is scattered about 2.7 times more by the earth's atmosphere as compared to low-pressure sodium lamps that emit at $589.3 \mathrm{~nm}$.Cool-white LEDs are not suitable for using at outdoor lighting near astronomical observatories.

The light is used in fully-shielded way to minimize Blue pollution in Luminanz technology.

\section{CONCLUSION}

The case study of lighting sources of an academic building is done keeping illumination constant. The light intensity, power savings, cost savings, initial cost are least in case of LED. Though LED also has some disadvantages, as blue hazards, blue pollution, light quality, area light source. Still it can be concluded that LED is the best option of all.

If further research for these problems is done and the problems can be eliminated and then LED would be the best lighting source for the time being.

\section{REFERENCES}

[1]. Light Emitting Diodes: The future of lighting, IEEMA Journal, Volume XXVII no. 10 October 2007

[2]. U.S. Department of Energy (2013) Energy efficiency of LEDs. Retrieved Oct 3, 2013, from http://apps1.eere.energy.gov/buildings/publications/pdfs/ssl/led_energy_efficiency.pdf

[3]. N. V. Vader, R. U. Patil, Energy Conservation In Electrical System, National conference on recent trends in Engineering \& Technology Organized by Agnel Polytechnic, Vashi in association with IIE ZENITH - 2009, 30 - 31st OCT -09

[4]. V. S. Verma, 'Energy Efficient Technologies use in India - An Overview 2004', Bureau of Energy Efficiency (BEE), 20 August 2004

[5]. A. N. Singh, J. Sharma, Energy Conservation in India: Challenges \& Achievements, International Journal of Mechanical and Industrial Engineering (IJMIE), Volume-1, Issue-3, 2012, ISSN No. $2231-6477$ 